\title{
Fabrication of various micropatterns by maskless micro-electrochemical texturing
}

\author{
Sandip Kunar* and Bijoy Bhattacharyya \\ Department of Production Engineering, Jadavpur University, Kolkata 700032, India
}

Received: 2 January 2019 / Accepted: 27 March 2019

\begin{abstract}
In this paper, an innovative and alternative concept of maskless micro-electrochemical texturing is exploited for the fabrication of simple and complex micropatterns. In this process, the tool is masked incorporated with the textured patterns and the workpiece has no mask. This research study concentrates on generation of simple micropattern, i.e. linear micropattern, and complex micropattern, i.e. cascade micropattern using maskless micro-electrochemical texturing method without repeated use of photolithography process. A single masked patterned tool with SU-8 2150 mask can produce many high-quality simple and complex micropatterns economically using this method. A well-planned experimental set-up consisting of electrochemical micromachining (EMM) cell, electrode fixtures, electrical connections and constricted vertical cross-flow electrolyte system has been designed and developed indigenously for carrying out the experiments. Influences of major influencing parameters, i.e. machining voltage, interelectrode gap, flow rate and machining time, are investigated on width overcut and machining depth of micropatterns. For higher machining accuracy, controlled depth and lower standard deviations, machining with lower machining time, lower voltage, lower interelectrode gap and higher flow rate is recommended. From the detailed experimental investigation, the best parametric combination are voltage of $8 \mathrm{~V}$, duty ratio of $30 \%$, pulse frequency of $15 \mathrm{kHz}$, electrolyte of $\mathrm{NaCl}$ $(0.34 \mathrm{M})+\mathrm{NaNO}_{3}(0.23 \mathrm{M})$, flow rate of $5.35 \mathrm{~m}^{3} / \mathrm{h}$, interelectrode gap of $50 \mu \mathrm{m}$ and machining time of $40 \mathrm{~s}$.
\end{abstract}

Keywords: Maskless micro-electrochemical texturing / micropatterns / linear / cascade / reused masked tool / width overcut / machining depth

\section{Introduction}

In the present scenario, the perception of surface phenomena, especially microsurface textures at a microand nanoscale, has played a significant role in many advance fields such as microelectronics, micromixtures, microcoolers, microreactors, optics, tribology, biology, aerospace, information technology, etc. Surface texturing method is used to define and modify the engineered surfaces for specific function. For the advancement of technology in the area of microfabrication, microsurface texture has performed many important functions economically in many microengineering applications such as sensors, biomedical, electronics, chemical microreactors, microelectromechanical systems, etc. [1].

Ghoshal and Bhattacharyya [2] have fabricated microchannels and taperless microslots are generated with reduced overcut, taper angle and corner deviation by EMM method using single microtool. Rathod et al. [3] have

\footnotetext{
* e-mail: sandip.sandip.kunar@gmail.com
}

investigated the effects of major EMM process parameters on width overcut, depth overcut, length overcut, profile depth, material removal rate, linearity of microgroove and surface finish during generation of microgrooves by a single microtool using EMM method. Zhang et al. [4] have produced a single microgroove having width of $30 \mu \mathrm{m}$ in situ fabricated electrochemical micromachining set-up using $8 \mu \mathrm{m}$ diameter tungsten tool electrode. Liu et al. [5] have utilized a developed electrochemical micromachining method to generate microcomplex structure with good shape and surface quality using ultrashort pulses. But these types of production processes are time-consuming and expensive due to one by one fabrication of microgroove, microchannel, etc. Schönenberger and Roy [6] have generated square patterns and linear profiles on copper substrate by electrochemical etching method. The depth of etched patterns is very low. Kunar et al. [7] have used the maskless EMM method to fabricate the complex shape of varactor micropattern. The generated microfeature is very complex. Byun et al. [8] have used the micro-ECM with microsecond pulses to fabricate the microdimple pattern using single microtool electrode. But this process is 
time-consuming and costly due to one by one hole fabrication by a single tool electrode. Yong et al. [9] have successfully applied electrochemical micromachining technology to fabricate complex microstructure with good shape precision and surface quality and the effect of some influential electrochemical process parameters such as electrode geometry features, feed rate, electrolyte composition and electrical parameters on machining quality is investigated. Sjöström and Su [10] have employed the electrochemical micromachining method to produce pit and groove structures at high rates using a simple tungsten carbide tool. Ghoshal and Bhattacharyya [11] have utilized the electrochemical micromachining method as a key micromachining technique to generate ultrasize components and microprofiles using single microtool. Jo et al. [12] have presented the application of micro-electrochemical machining for micromachining of internal microfeatures by controlling pulse conditions and machining time. These types of fabrication processes using a single microtool are time-consuming and costly. Many micromachining technologies are employed to generate diverse simple and complex surface textures such as electrical discharge machining, mechanical machining, chemical etching, laser beam machining, through-mask EMM, etc. However, most of the methods are not appropriate to fulfil the needs of modern industries for production of economical microsurface textures. These processes have post-machined cleaning cost, more rework and lower productivity. So, maskless micro-electrochemical texturing method is an important alternative method for generating dissimilar simple and complex microstructures due to its imperative advantages, including higher machining rate, bright surface finish, reusability of a single patterned tool, no heat-affected zone, burr-free surface and no tool wear.

An innovative and alternative concept of maskless micro-electrochemical texturing is proposed for fabrication of simple and complex micropatterns on stainless steel. In this research study, the indigenous development of maskless micro-electrochemical texturing set-up is concentrated for conducting the experiments using developed and constricted vertical cross-flow electrolyte supply system for the generation of simple and complex micropatterns. One textured patterned tool with SU-8 2150 mask can generate many linear and cascade micropatterns at a low cost and in short time by using this method without repeated use of photolithography process. The effect of major process parameters such as voltage, interelectrode gap, electrolyte flow rate and machining time on width overcut and machining depth is studied using this process. An attempt has also been made to present the analysis of micrographs for getting suitable parametric combination.

\section{Experimental procedure}

The developed maskless micro-electrochemical texturing set-up has been utilized for carrying out the experiments for generation of linear and cascade micropatterns, as shown in Figure 1. The experimental set-up consists of EMM cell, electrode fixtures, power supply connections and electrolyte circulation system. EMM cell has

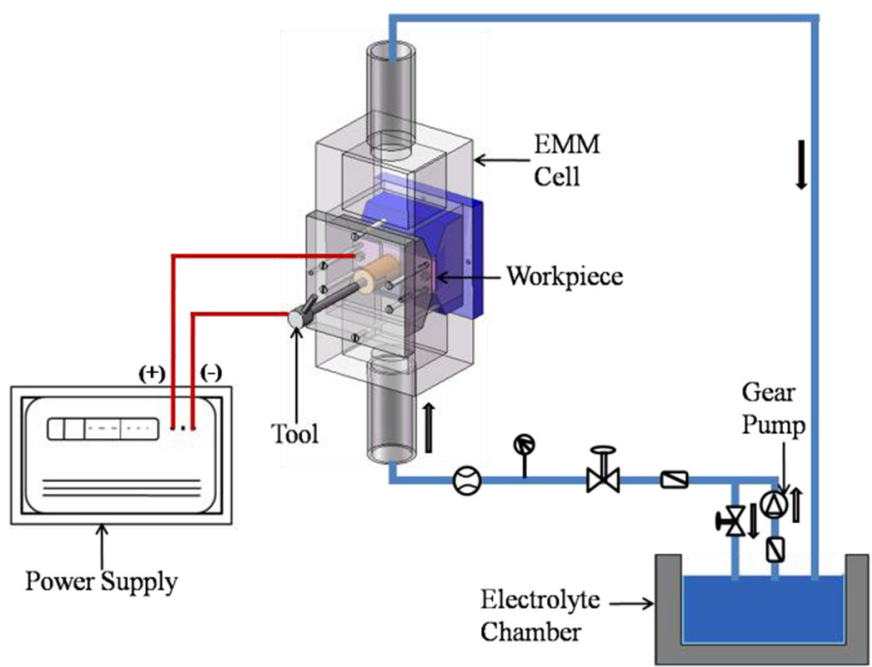

Fig. 1. Maskless micro-electrochemical texturing set-up.

electrode-holding devices, electrical connection system and constricted vertical cross-flow electrolyte system. EMM cell with electrode fixtures are made of Perspex to avoid corrosion. The inlet and outlet segments of EMM cell are made of stainless steel to protect from oxidation. Covered nature of EMM cell protects from the flushing of electrolyte, and electrolyte is circulated through the tool and workpiece and return to the reservoir for recirculation purpose. It has transparent Perspex blocks in which the machining operation can be observed. Workpiece and tool fixtures with vertical cross-flow electrolyte system are developed inside the machining cell. Figure 2 represents the developed maskless EMM cell with tool and job fixtures. Gear pump is used for EMM system to pump clean electrolyte from the reservoir tank and it is supplied in the micromachining zone at desired pressure through inlet port. Then it is passed through the workpiece and tool. The used electrolyte goes to the electrolyte chamber through the outlet port. The important feature in electrolyte circulation system is vertical cross-flow electrolyte supply system, in which the flow is parallel to the tool and workpiece and flows vertically from downward to upward directions. Vertical cross-flow electrolyte system is more suitable for good flushing conditions in the micromachining zone because this flow creates additional back pressure in the machining zone, which is more suitable for sludge removal during machining and keeps the clean condition in the micromachining zone. The pulsed DC power supply is built with function generator and it has compact size, protection functions and ultrafast response. The pulse current is supplied to electrodes at constant voltage mode using square waveform.

To fabricate the masked patterned tool, SU-8 2150 negative photoresist (MicroChem, USA) on stainless steel wafer is used. This mask has enough strength to endure the high flow rate during experiments. Subsequent UV exposure system is used to fabricate linear and cascade micropatterns on stainless steel wafers using SU-8 2150 mask. The thickness of SU-8 2150 mask on wafers is 


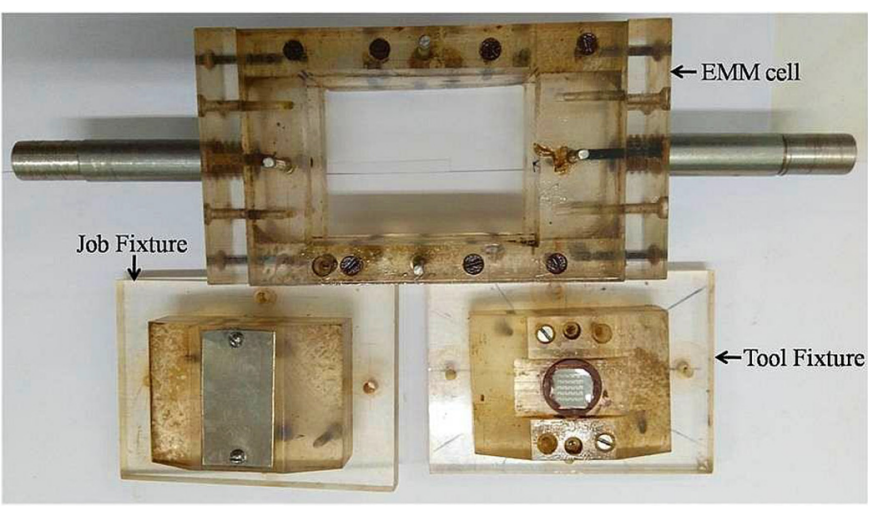

Fig. 2. Developed maskless EMM cell with tool and workpiece fixtures.

$220 \mu \mathrm{m}$. The width of fabricated linear micropattern is $460 \mu \mathrm{m}$ and the gap between two consecutive linear slots is $1000 \mu \mathrm{m}$. The width of fabricated complex cascade micropattern is $435 \mu \mathrm{m}$. After preparing of these samples, a small copper rod is attached with stainless wafers using adhesive silver paste and then precision micrometer is connected with copper rod for interelectrode gap settings. Then the excess area of copper rod is insulated with non-conductive enamel to protect from current density distribution on workpiece.

For proper engineering applicability of maskless microelectrochemical texturing set-up, the significant EMM parameters are finalized on the basis of extensive trial experiments and final experiments are carried out by varying one parameter at a time with other constant parameter setting on the basis of good experimental results. Influences of significant process variables are investigated on textured characteristics, i.e. width overcut and machining depth of generated linear and cascade micropatterns on stainless steel (SS-304). The ranges of applied voltage, interelectrode gap, electrolyte flow rate and machining time are $8-14 \mathrm{~V}, 50-200 \mu \mathrm{m}, 2.35-5.35 \mathrm{~m}^{3} / \mathrm{h}$ and $40-70 \mathrm{~s}$, respectively, and other factors, i.e. duty ratio of $30 \%$ and pulse frequency of $15 \mathrm{kHz}$, are kept constant. At first, the set of experiments for various voltages are carried out keeping other parameters constant and then the most suitable applied voltage is selected for the next level of experiments due to overall uniform machined characteristics and proper geometrical shape. Thereafter, other set of experiments for various interelectrode gaps are performed taking the selected applied voltage and other constant parameters. The proper interelectrode gap is preferred for the next level of experiments due to almost uniform surface characteristics and good geometrical shape. Other set of experiments for machining time and flow rate are carried out in a similar way, respectively. Mixed electrolyte of $\mathrm{NaCl}(0.34 \mathrm{M})+\mathrm{NaNO}_{3}(0.23 \mathrm{M})$ is selected for better machining because sodium chloride has higher material removal rate and sodium nitrate has higher machining accuracy. Each experiment is repeated five times and average values of each machined response are calculated. Interelectrode gap is the distance between tool and workpiece. Width overcut is the mean width of the profiles.

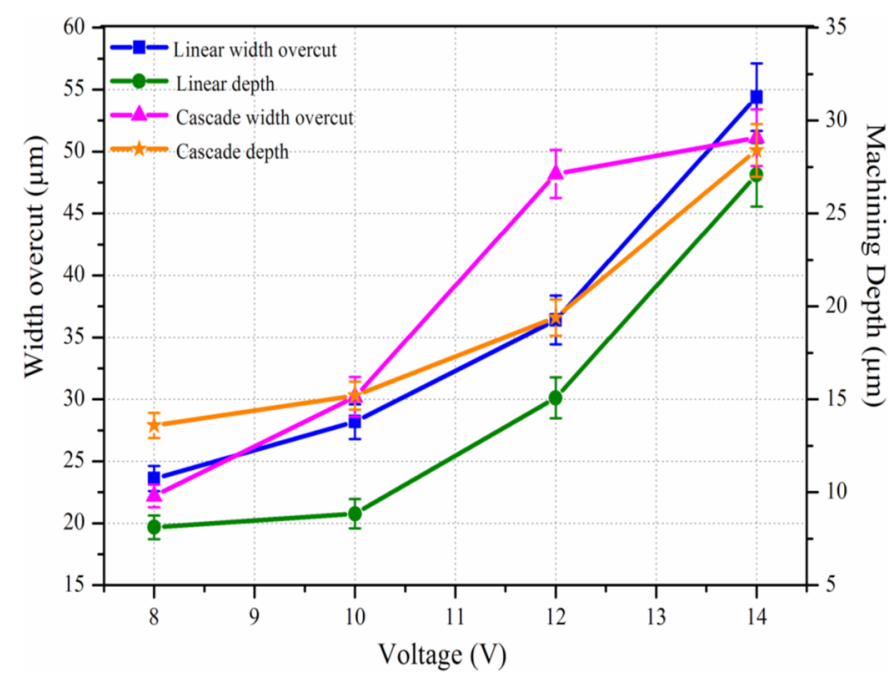

Fig. 3. Effect of machining voltage on width overcut and depth.

The standard deviations of machined responses are calculated to find out the quality of simple and complex micropatterns. The textured characteristics are examined with Optical Microscope (Leica EZ4D, Germany), ThreeDimensional Non-Contact Profilometer (CCI Sunstar, Taylor Hobson Ltd., UK) and Scanning Electron Microscope (JEOL, JSM-6360, Japan).

\section{Results and discussion}

Effects of important EMM process parameters, namely applied voltage, interelectrode gap, electrolyte flow rate and machining time on machined criteria, i.e. width overcut and machining depth of linear and cascade micropatterns, are investigated in the form of graphs.

\subsection{Influences of EMM parameters on micropatterned characteristics}

Experimental investigations are conducted to investigate the effect of applied voltage on width overcut and machining depth at a particular machining conditions, i.e. interelectrode gap of $50 \mu \mathrm{m}$, flow rate of $5.35 \mathrm{~m}^{3} / \mathrm{h}$ and machining time of 40 s using SU-8 2150 mask, as shown in Figure 3. It is seen that the width overcut of linear and cascade micropatterns increases with increasing machining voltage. Higher voltage increases the stray current effect and current density. As a result, the machining rate is faster from the periphery of micropatterns and the width overcut of micropatterns easily increases with increase in machining voltage. In addition, the accumulation of electrolysis products and gas bubbles increases with the increase in applied voltage in the micromachining zone, which reduces the machining accuracy of micropatterns. The machining depth of linear and cascade micropatterns increases with increase in applied voltage. With increasing voltage, the machining current and current density increase. The increasing current and current density 


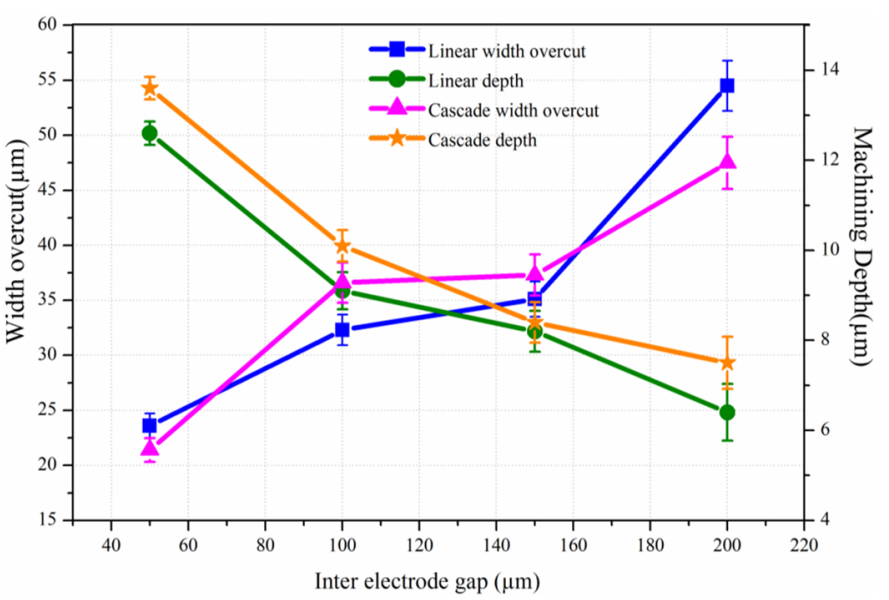

Fig. 4. Effect of interelectrode gap on width overcut and depth.

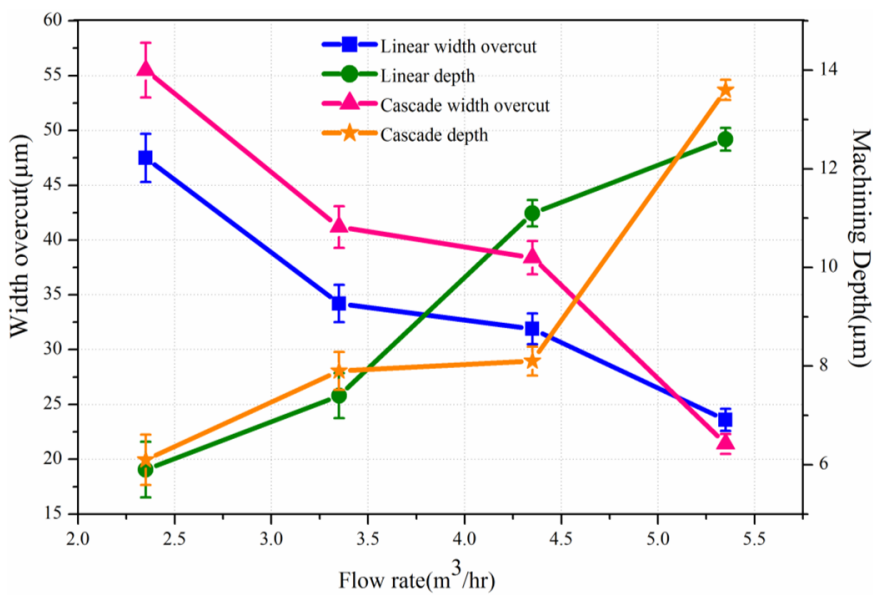

Fig. 5. Effect of flow rate on width overcut and depth. increase the machining localization across the machining zone. Lower standard deviations in width overcut and machining depth are observed at low voltage due to controlled etching and higher standard deviations are shown in higher voltage due to uncontrolled anodic dissolution. Lower machining voltage is recommended for better machining accuracy, controlled depth and lower standard deviation. At the machining voltage $<8 \mathrm{~V}$, the micropatterns are not machined properly and at the machining voltage $>14 \mathrm{~V}$, the generated micropatterns are non-uniform in shape and size.

Experimentation is carried out to study the influence of interelectrode gap on width overcut and machining depth with other constant parameter setting, i.e. voltage of $8 \mathrm{~V}$, flow rate of $5.35 \mathrm{~m}^{3} / \mathrm{h}$ and machining time of $40 \mathrm{~s}$ using SU-8 2150 mask, as shown in Figure 4. The most influencing EMM parameter in maskless micro-electrochemical texturing method is interelectrode gap for generating the better simple micropattern, i.e. linear micropattern, and complex micropattern, i.e. cascade micropattern with higher machining accuracy. Lower interelectrode gap between tool and workpiece can increase the machining accuracy of the micropatterns during machining because controlled current flows between tool and workpiece. Higher interelectrode gap between tool and workpiece deteriorates the machining accuracy of the micropatterns during machining because the stray current flow is uncontrolled between tool and workpiece. The width overcut of linear micropattern and cascade micropattern increases with increasing interelectrode gap. In lower interelectrode gap, the fabricated micropatterns are regular in shape and size due to reduced stray current effect for controlled current density distribution. In the higher interelectrode gap, the width overcut of micropatterns is higher due to higher stray current effect for non-uniform current flux distribution. The machining depth of micropatterns decreases with increasing interelectrode gap because the machining localization decreases due to higher ohmic resistance and uncontrolled current flux distribution. Lower standard deviations in width overcut and machining depth are observed at lower interelectrode gap due to controlled etching for controlled current density and higher standard deviations are revealed in higher voltage due to uncontrolled anodic dissolution for non-uniform current density distribution. For precise micropatterns, machining with lower interelectrode gap is recommended for lower width overcut, controlled depth and lower standard deviation. At the interelectrode gap $<50 \mu \mathrm{m}$, the micropatterns are not machined properly and at the interelectrode gap $>200 \mu \mathrm{m}$, the generated micropatterns are irregular in shape and size.

Figure 5 represents the effect of flow rate on width overcut and machining depth of linear and cascade micropatterns. Micropatterns are fabricated by varying flow rate from 2.35 to $5.35 \mathrm{~m}^{3} / \mathrm{h}$ with other constant parameters, i.e. voltage of $8 \mathrm{~V}$, interelectrode gap of $50 \mu \mathrm{m}$ and machining time of $40 \mathrm{~s}$, using SU-8 2150 mask. The width overcut of linear and cascade micropatterns decreases with increasing flow rate due to reduced stray current effect for faster removal of sludges and gas bubbles from the micromachining zone. The machining depth of micropatterns increases with increasing flow rate because the machining localization effect is higher due to higher current density. Lower standard deviations in width overcut and machining depth are observed at higher flow rate due to uniform etching and higher standard deviations are shown in lower flow rate due to uncontrolled anodic dissolution. To obtain proper geometrical shape of micropatterns, machining with higher flow rate is recommended for higher machining accuracy, controlled depth and lower standard deviation. At the flow rate $<2.35 \mathrm{~m}^{3} / \mathrm{h}$, the micropatterns are not formed properly. At the flow rate $>5.35 \mathrm{~m}^{3 /} \mathrm{h}$, the micropatterns reduce the geometrical shape.

Figure 6 shows the influence of machining time on width overcut and machining depth of linear and cascade micropatterns and other parameters are kept constant, i.e. voltage of $8 \mathrm{~V}$, flow rate of $5.35 \mathrm{~m}^{3} / \mathrm{h}$ and interelectrode gap of $50 \mu \mathrm{m}$. The width overcut of linear and cascade micropatterns increases with increasing machining time because higher machining time increases current density resulting in higher stray current effect. Higher stray current removes more material from the margin of 
micropatterns. The machining depth increases with increasing machining time due to higher machining localization. Lower standard deviations in width overcut and machining depth are observed at lower machining time due to uniform etching and higher standard deviations are shown in higher machining time due to lower machining localization. For better shape of micropatterns, machining with lower machining time is suggested for higher machining accuracy, controlled depth and lower standard deviation. At the machining time $<40 \mathrm{~s}$, the micropatterns are not formed precisely. At the machining time $>70 \mathrm{~s}$, the micropatterns deteriorate the proper geometrical shape.

\subsection{Analysis of micrographs}

The textured cathode tool with SU-8 2150 mask having linear micropattern has more potentiality for generating numerous linear micropatterns. This masked tool is fabricated by photolithography process. This single masked tool can be reused more than 26 times to carry out the experiments using maskless micro-electrochemical

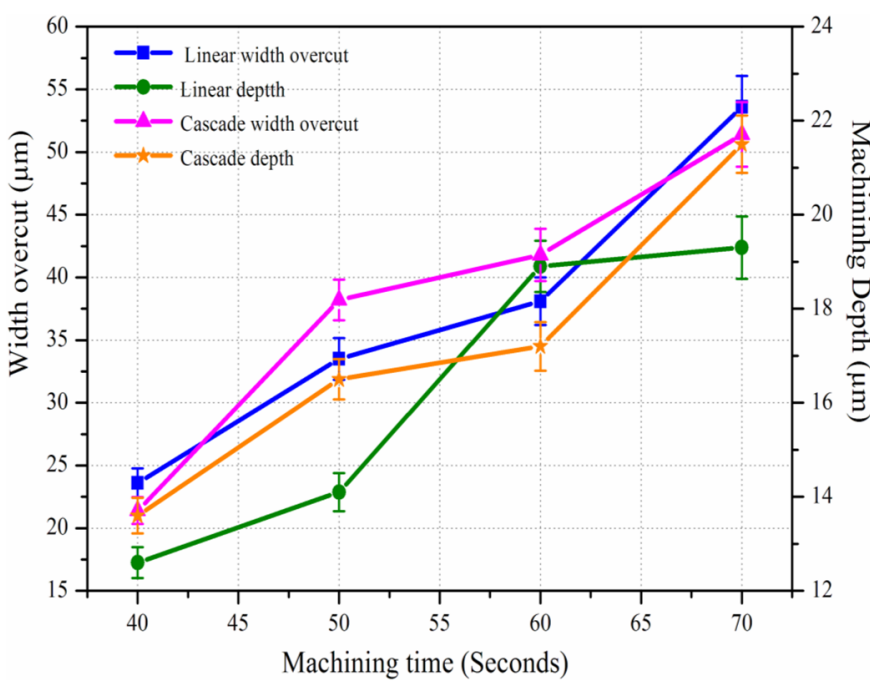

Fig. 6. Effect of machining time on width overcut and depth. texturing process without repeated use of photolithography process. It does not distort during experiments. Figure 7 a shows the simple micropattern, i.e. linear micropattern of SU-8 2150 mask before machining. It is reused many times because it has faster drying capacity, ability to adhere with substrates, high structural strength, etc. Figure $7 \mathrm{~b}$ shows the patterned mask of linear micropattern after being used 26 times. These results recommend that this mask can generate many machined samples of linear micropatterns with better geometrical shape without distortion of mask. But through-mask electrochemical micromachining is a time-consuming and expensive process for fabrication of many simple linear micropatterns due to individual masking of each workpiece before machining, in which photolithography is always required for masking on each workpiece before machining. So, maskless microelectrochemical texturing process is an alternative concept of electrochemical micromachining process through-mask for fabricating of simple micropatterns economically without repeated use of photolithography process.

Figure 8a shows the SEM image of generated linear micropattern and it is fabricated at a particular parametric combination such as voltage of $8 \mathrm{~V}$, duty ratio of $30 \%$, pulse frequency of $15 \mathrm{kHz}$, electrolyte of $\mathrm{NaCl}(0.34 \mathrm{M})+\mathrm{NaNO}_{3}$ $(0.23 \mathrm{M})$, flow rate of $5.35 \mathrm{~m}^{3} / \mathrm{h}$, interelectrode gap of $50 \mu \mathrm{m}$ and machining time of $40 \mathrm{~s}$ using SU-8 2150 mask. The generated linear micropattern has proper geometrical shape and size because the controlled machining takes place for uniform current density distribution. As a result, the material removal is quite uniform from the margin of linear micropattern. The distribution of machining depth is uniform across the machining zone because higher machining localization takes place for controlled current density distribution. The mean width overcut and mean machining depth of linear micropattern are 23.6 and $12.6 \mu \mathrm{m}$, respectively. Figure $8 \mathrm{~b}$ shows $3 \mathrm{D}$ view of the segment of a single microslot with the details of depth measurement having height difference of $44.5 \mu \mathrm{m}$. The depth has been measured by the 3D Non-Contact Profilometer (CCI Sunstar, Taylor Hobson Ltd, UK).

Complex micropattern, namely cascade micropatterns, are fabricated by maskless micro-electrochemical texturing process using SU-8 2150 negative photoresist. Figure 9a

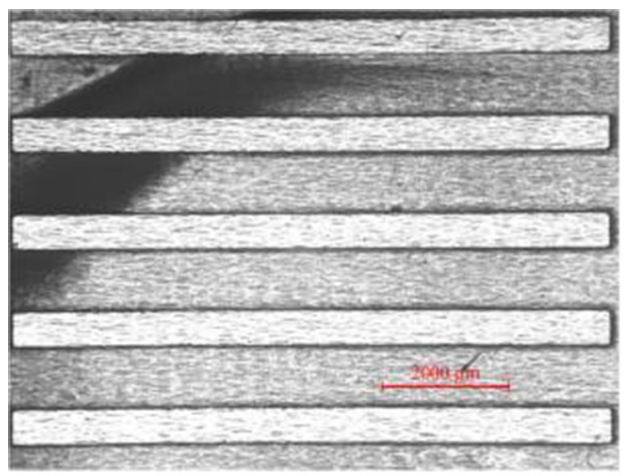

(a)

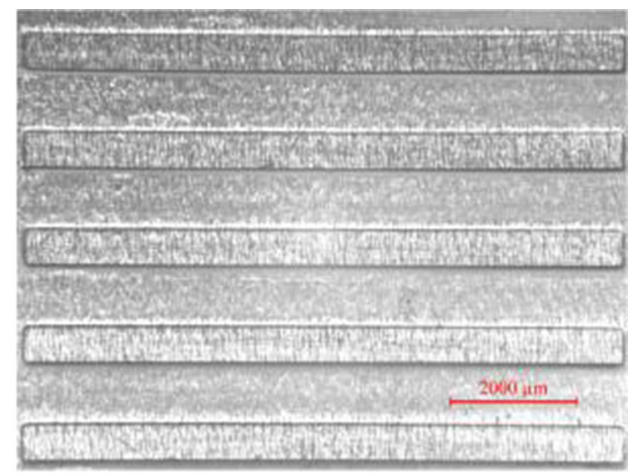

(b)

Fig. 7. (a) Unused mask and (b) mask after being used 26 times. 


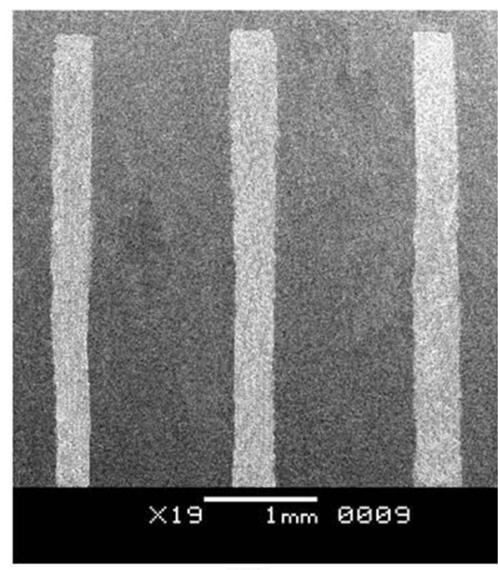

(a)
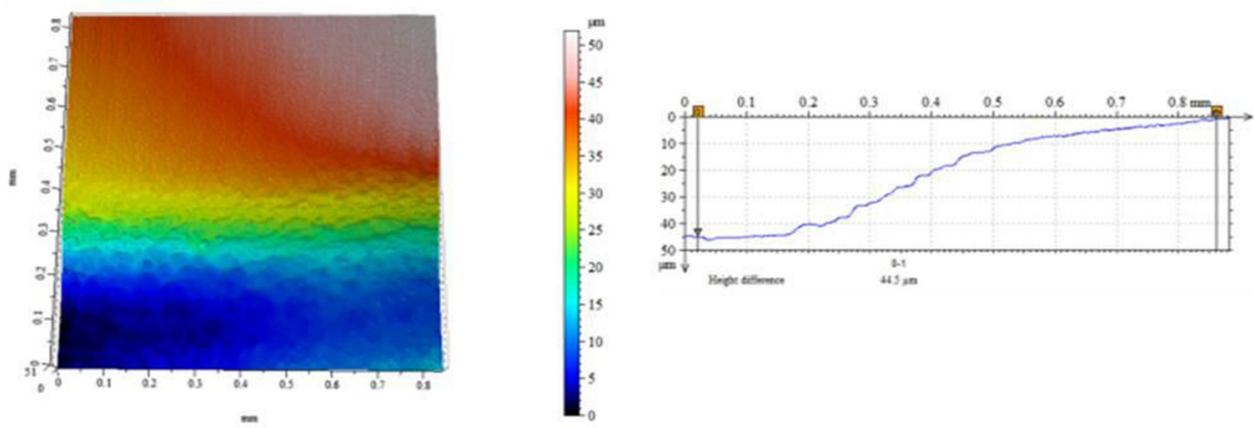

(b)

Fig. 8. (a) Regular linear micropattern. (b) $3 \mathrm{D}$ view and depth profile of a segment of regular linear micropattern.

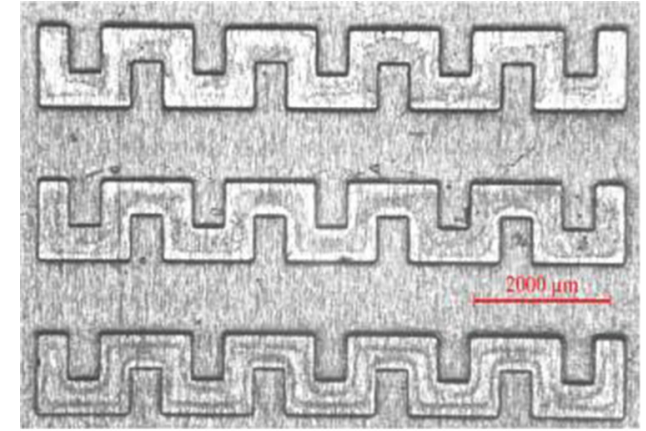

(a)

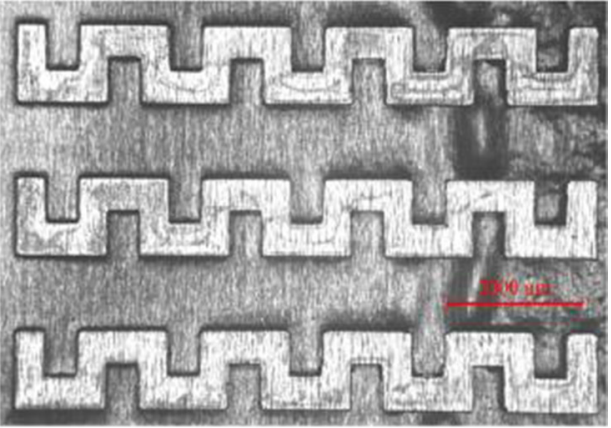

(b)

Fig. 9. (a) Unused mask and (b) mask after being used 26 times.

shows the cascade micropattern using SU-8 2150 mask on stainless steel wafer before machining. It is reused over 26 times for fabricating cascade micropatterns without repeated use of photolithography process. Figure $9 \mathrm{~b}$ shows the cascade micropatterned mask after being used twenty six times. It is proved that this mask has capability to fabricate many complex micropatterned samples with good geometrical shape. This mask does not deform after being used 26 times. So, it has no deformation in the patterned mask after generating of many samples. But photolithography process is necessary in electrochemical micromachining through-mask before machining because individual masking is required for each workpiece before machining. This process is a time-consuming and costly process for fabrication of many complex cascade micropatterns. So, maskless micro-electrochemical texturing process is an alternative concept of electrochemical micromachining through-mask process for generating complex micropatterns in short time and at a low cost without repeated use of photolithography process. 


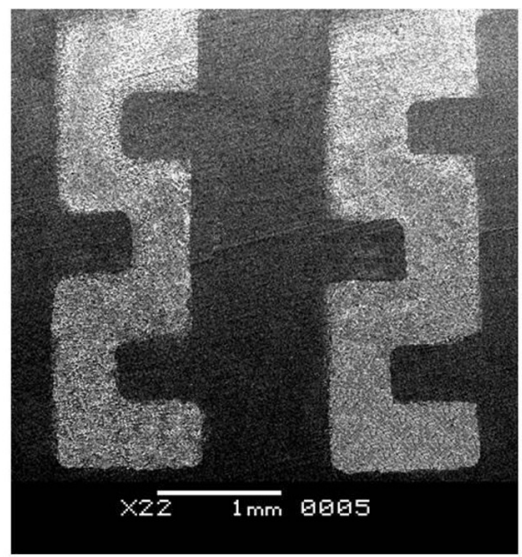

(a)
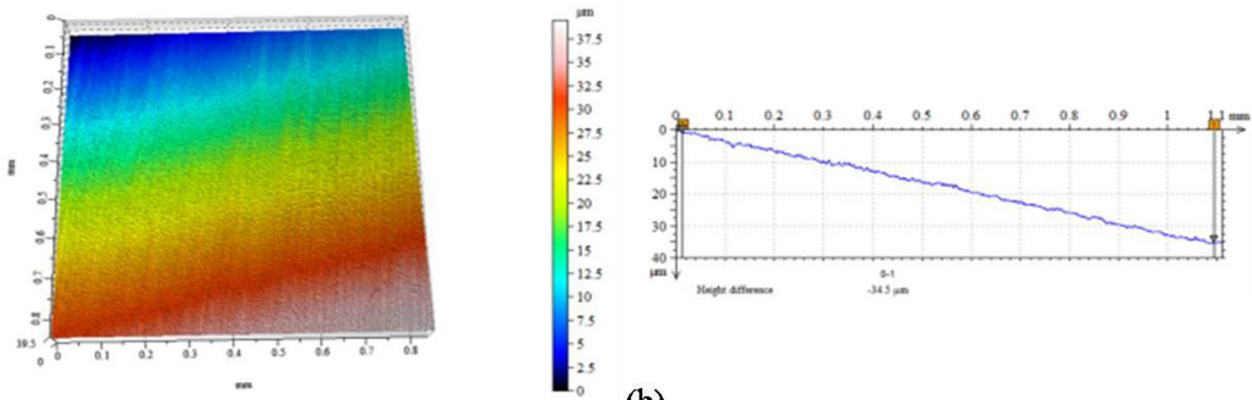

(b)

Fig. 10. (a) Regular cascade micropattern. (b) 3D view and depth profile of a segment of regular cascade micropattern.

The SEM image of generated complex cascade micropattern has been shown in Figure 10a and it is generated at a particular parametric setting, namely voltage of $8 \mathrm{~V}$, duty ratio of $30 \%$, pulse frequency of $15 \mathrm{kHz}$, electrolyte of $\mathrm{NaCl}(0.34 \mathrm{M})+\mathrm{NaNO}_{3}(0.23 \mathrm{M})$, flow rate of $5.35 \mathrm{~m}^{3} / \mathrm{h}$, interelectrode gap of $50 \mu \mathrm{m}$ and machining time of $40 \mathrm{~s}$ using SU-8 2150 mask. The fabricated complex micropattern, i.e. cascade micropattern, has proper geometrical shape due to controlled machining. The distribution of machining depth is also controlled for higher machining localization due to uniform current density distribution. The mean width overcut and mean machining depth of generated complex cascade micropattern are 21.4 and $13.6 \mu \mathrm{m}$, respectively. Figure $10 \mathrm{~b}$ shows a $3 \mathrm{D}$ image and the details of depth measurement of a segment of cascade micropattern with a height difference of $34.5 \mu \mathrm{m}$.

\section{Conclusions}

Generation of simple and complex micropatterns on stainless steel has been demonstrated utilizing maskless micro-electrochemical texturing process. This article represents the influence of major EMM process parameters, i.e. applied voltage, interelectrode gap, flow rate and machining time on width overcut and machining depth of generated simple and complex micropatterns. Experiments have been carried out by varying one EMM parameter at a time by keeping other process parameters constant using patterned cathode tools. Various research findings of these experiments are as follows:

- Maskless micro-electrochemical texturing process is the unique and alternative concept of generation of linear and cascade micropatterns without repeated use of photolithography process utilizing indigenously developed maskless EMM set-up having EMM cell and constricted vertical cross-flow system in short time and at a low cost.

- A single textured cathode tool using SU-8 2150 mask containing linear and cascade micropatterns can fabricate many machined samples more than 26 times with controlled shape and size without repeated use of photolithography process.

- For higher machining accuracy, controlled depth and lower standard deviations, machining with lower interelectrode gap, lower machining time, lower voltage and higher flow rate is recommended.

From the detailed analysis of micrographs of simple and complex micropatterns, the best parametric combination are voltage of $8 \mathrm{~V}$, duty ratio of $30 \%$, pulse frequency of $15 \mathrm{kHz}$, electrolyte of $\mathrm{NaCl}(0.34 \mathrm{M})+\mathrm{NaNO}_{3}(0.23 \mathrm{M})$, flow rate of $5.35 \mathrm{~m}^{3} / \mathrm{h}$, interelectrode gap of $50 \mu \mathrm{m}$ and machining time of $40 \mathrm{~s}$ using SU-8 2150 mask.

Maskless micro-electrochemical texturing process will be very helpful for fabrication of various micropatterns with different shapes and sizes on different conductive materials. Hence, for better geometrical shape and size, the design of a proper patterned tool and tool movement strategy need to be improved further. 


\section{References}

1. A.A.G. Bruzzone, H.L. Costa, P.M. Lonardo, D.A. Lucca, Advances in engineered surfaces for functional performance, CIRP Ann. Manuf. Technol. 57 (2008) 750-769

2. B. Ghoshal, B. Bhattacharyya, Generation of microfeatures on stainless steel by electrochemical micromachining, Int. J. Adv. Manuf. Technol. 76 (2015) 39-50

3. V. Rathod, B. Doloi, B. Bhattacharyya, Experimental investigations into machining accuracy and surface roughness of microgrooves fabricated by electrochemical micromachining, J. Eng. Manuf. 229 (2015) 1-22

4. Z. Zhang, Y. Wang, F. Chen, W. Mao, A micromachining system based on electrochemical dissolution of material, Russ. J. Electrochem. 47 (2011) 819-824

5. Y. Liu, D. Zhu, Y. Zeng, S. Huang, H. Yu, Experimental investigation on complex structures machining by electrochemical micromachining technology, Chin. J. Aeronaut. 23 (2010) 578-584

6. I. Schönenberger, S. Roy, Microscale pattern transfer without photolithography of substrates, Electrochim. Acta 51 (2005) 809-819
7. S. Kunar, S. Mahata, B. Bhattacharyya, Influence of electrochemical micromachining process parameters during fabrication of varactor micropattern, Int. J. Adv. Manuf. Technol. 96 (2018) 411-427

8. J.W. Byun, H.S. Shin, M. H. Kwon, B. H. Kim, C. N. Chu, Surface texturing by micro ECM for friction reduction, Int. J. Precis. Eng. Manuf. 11 (2010) 747-753

9. L. Yong, Z. Di, Z. Yongbin, H. Shaofu, Y. Hongbing, Experimental investigation on complex structures machining by electrochemical micromachining technology, Chinese J. Aeronaut. 23 (2010) 578-584

10. T Sjöström, B. Su, Micropatterning of titanium surfaces using electrochemical micromachining with an ethylene glycol electrolyte, Mater. Lett. 65 (2011) 3489-3492

11. B. Ghoshal, B. Bhattacharyya, Investigation on profile of microchannel generated by electrochemical micromachining, J. Mater. Process. Technol. 222 (2015) 410-421

12. C.H. Jo, B.H. Kim, C.N. Chu, Micro electrochemical machining for complex internal micro features, CIRP Ann. Manuf. Technol. 58 (2009) 181-184

Cite this article as: S. Kunar, B. Bhattacharyya, Fabrication of various micropatterns by maskless micro-electrochemical texturing, Manufacturing Rev. 6, 6 (2019) 